\title{
Brucellose-Erkrankungen in Deutschland
}

\author{
Von G. Rasch, I. Schöneberg, L. Apitzsch und U. Menzel
}

\section{Einführung}

Brucellose-Erkrankungen des Menschen gehören zu den nach $\$ 3$ BSeuchG meldepflichtigen Infektionskrankheiten. Sie haben, nach der Zahl der jährlich registrierten Erkrankungen, an den Infektionskrankheiten in Deutschland insgesamt nur einen geringen Anteil.

Wurden Brucellosen in früheren Jahren vorwiegend durch Kontakte $\mathrm{zu}$ erkrankten Tieren im eigenen Land erworben, so spielen heute Übertragungen durch Lebensmittel tierischer Herkunft eine wichtige Rolle. Dies steht in engem Zusammenhang mit der Sanierung der deutschen Tierbestände. In Deutschland gezüchtete Rinder sind weiterhin Brucellose-frei. Beobachtete Neuausbrüche bei Rindern durch Brucella abortus, Biotyp 3 sind auf Tierimporte zurückzuführen. Gegenwärtig besteht kein direkter Zusammenhang mehr zwischen den Erkrankungen bei Tieren und beim Menschen.

Anknüpfend an die Darstellungen zur Situation der Brucellose aus der Sicht der Biotypisierung von Brucellen am BGA/BgVV im Februar-Heft 1996 des Bundesgesundheitsblattes [1] soll im folgenden speziell auf die BrucelloseErkrankungen des Menschen eingegangen werden.

\section{Brucellose-Erkrankungen und -Sterbefälle 1947-1995}

Brucellose-Erkrankungen, für die Angaben ab 1947 (im alten Bundesgebiet) und für die DDR ab 1951 vorliegen, erreichten in der Mitte der fünfziger Jahre im alten Bundesgebiet und in den sechziger Jahren auf dem Gebiet der DDR ihre größte Verbreitung (Abb. 1). In der Folge kam es in beiden Gebieten zu einem deutlichen Rückgang der Erkrankungszahlen (Anfang der sechziger Jahre bzw. ab 1975). Seit Beginn der achtziger Jahre liegt die Zahl der gemeldeten Erkrankungen in jedem Jahr insgesamt unter 100 (Tab. 1). Eine Ausnahnre stellt das Jahr 1983 dar, in dem, wie auch schon im Jahre 1982, kleinere Epidemien beobachtet wurden [2].

In den letzten zehn Jahren lag die Zahl der jährlich vom Statistischen Bundes-
Tabelle 1: Erkrankungen und Sterbefälle an Brucellose in Deutschland (nach $[3,4]$ )

\begin{tabular}{|c|c|c|c|c|}
\hline Jahr & $\begin{array}{l}\text { Erkrankungen } \\
\text { West/gesamt }\end{array}$ & $\begin{array}{l}\text { Sterbefälle } \\
\text { West/gesamt }\end{array}$ & $\begin{array}{l}\text { Erkrankungen } \\
\text { Ost (bis 1990) }\end{array}$ & $\begin{array}{c}\text { Sterbefälle } \\
\text { Ost (bis 1990) }\end{array}$ \\
\hline 1980 & 81 & 1 & 15 & \\
\hline 1981 & 59 & & 2 & \\
\hline 1982 & 82 & & 3 & \\
\hline 1983 & 141 & & & \\
\hline 1984 & 59 & & & \\
\hline 1985 & 35 & 2 & 1 & \\
\hline 1986 & 39 & & & \\
\hline 1987 & 34 & & & \\
\hline 1988 & 34 & 1 & 1 & \\
\hline 1989 & 23 & & 3 & \\
\hline 1990 & 24 & & 4 & \\
\hline 1991 & 27 & 1 & & \\
\hline 1992 & 23 & & & \\
\hline 1993 & 21 & 2 & & \\
\hline 1994 & 28 & 1 & & \\
\hline 1995 & 35 & 1 & & \\
\hline
\end{tabular}

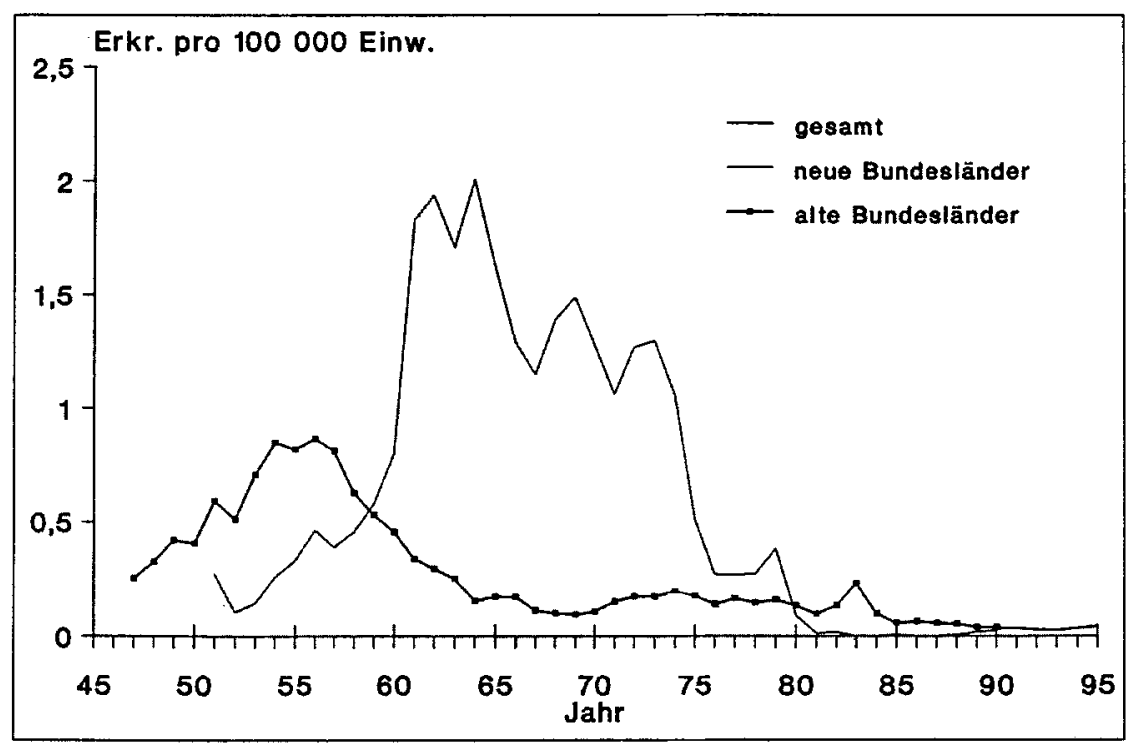

Abbildung 1: Brucellose in Deutschland - jährliche Inzidenzraten. 
amt registrierten Brucellose-Erkrankungen zwischen 21 und 35 [3]. Sterbefälle infolge Brucellose treten nur selten auf. Entsprechend der Todesursachenstatistik sind im Zeitraum der letzten 20 Jahre insgesamt elf Patienten an den Folgen dieser Erkrankung gestorben [4]

\section{Differenzierte Erfassung von Brucel-} lose-Erkrankungen

Brucellose-Erkrankungen wurden in den zurückliegenden Jahren im alten Bundesgebiet in unterschiedlicher Weise erfaßt. So galt in den Jahren bis 1961 die Meldepflicht nur für die Bang sche Krankheit (verursacht durch $\mathrm{Br}$. abortus). Veränderungen in den Festlegungen des BSeuchG ermöglichten für die Folgejahre (bis 1979) eine nach Erregerarten differenzierte Erfassung der Brucellosen (Bangsche Krankheit, Maltafieber, übrige Formen). Durch die Erweiterung der Meldepflicht um das Maltafieber (Br. melitensis) und um die Suis-Brucellose (Br. suis) kam es jedoch zu keinem merklichen Anstieg der Erkrankungszahlen an Brucellose insgesamt. Das führte zu der Schlußfolgerung, daß sowohl das Maltafieber als auch die Schweinebrucellose in diesem Zeitraum in Deutschland keine große Rolle spielten [5].

$\mathrm{Da}$ die gesonderte Meldepflicht für Mal tafieber und übrige Formen der Brucellose keine wichtigen epidemiologischen Erkenntnisse erbracht hatte $[6,7]$, entfiel diese ätiologische Unterteilung der Brucellose mit dem Vierten Gesetz zur Änderung des BSeuchG zum 1. 1. 1980. Seither sind alle Erkrankungs- und Sterbefälle, unabhängig von Erkrankungsform und Erregerart, unter der Kategorie »Brucellose« meldepflichtig.

Allein auf der Grundlage der im Rahmen der Meldepflicht erhobenen Daten sind detaillierte Aussagen zum verursachenden Erreger, zur vermuteten Infektionsursache, zum Herkunftsland usw. zum gegenwärtigen Zeitpunkt nicht möglich.

\section{Sondererhebungen}

Ausgehend von einem in ersten Halbjahr 1995 aus Malta berichteten Ausbruch an Brucellose (verursacht durch Weichkäse) mit insgesamt 135 Erkrankungen und einem Todesfall sowie zwei von dort nach England eingeschleppten Erkrankungsfällen mit Nachweis von $\mathrm{Br}$. melitensis [8] erhob sich die Frage, ob auch unter den in Deutschland regi-

Tabelle 2: Nachgewiesene Brucella-Arten 1995

\begin{tabular}{c|c|c|c|c|c}
\hline Br. abortus & Br. melitensis & Br. ab./mel. & Br. (ohne Differ.) & ohne Angabe & Summe \\
\hline 8 & 15 & 4 & 1 & 6 & 34 \\
\hline $23 \%$ & $44 \%$ & $12 \%$ & $3 \%$ & $18 \%$ & $100 \%$ \\
\hline
\end{tabular}

strierten Erkrankungsfällen Importfälle aus Malta zu verzeichnen sind bzw. welcher Herkunft Brucellose-Erkrankungen in Deutschland sind.

Um nähere Angaben zur Infektionsursache und zu den Erregerarten der Brucellose-Erkrankungen zu erhalten, wurde ab dem Jahr 1995 mit einer Einzelfall-Erfassung mit Hilfe eines speziel entworfenen Erhebungsbogens begonnen (Abb. 2). Dieser Bogen befindet sich gegenwärtig noch in der praktischen $\mathrm{Er}$ probung und wird in absehbarer Zeit auch eine andere, ansprechendere Gestaltung erhalten.

Der Erhebungsbogen wurde den $\mathrm{Ge}$ sundheitsämtern nach erfolgter Meldung einer Brucellose-Erkrankung, entsprechend $\$ 3$ BSeuchG, mit der Bitte um möglichst vollständige Angaben zugesandt. Die Resonanz auf unsere Bitte zeigte eine große Bereitschaft der Kolleginnen und Kollegen, unser Anliegen zu unterstützen. An dieser Stelle se allen beteiligten Mitarbeitern der Gesundheitsämter, aber auch niedergelassenen Ärzten, Mitarbeitern von Krankenhäusern usw. herzlich gedankt, die durch ihre Mitarbeit und Unterstützung diese Sondererhebungen möglich machen.

\section{Ergebnisse der Sondererhebungen}

Zum gegenwärtigen Zeitpunkt liegen für 34 der dem RKI im Jahre 1995 insgesamt gemeldeten 36 Brucellose-Erkrankungen detaillierte Angaben vor. Die Angaben auf den uns zugesandten 34 Erhebungsbögen wurden in die nachfol- gend aufgeführten Auswertungen einbezogen.

Bei der Zahl der im Jahre 1995 registrierten Brucellose-Erkrankungen ergibt sich eine Diskrepanz zu den Daten des Statistischen Bundesamtes, die 35 Brucellosen ausweisen [3].

\section{Brucella-Arten}

Die Zuordnung der Erregerarten erfolgte auf der Grundlage eines durchgeführten Erregernachweises (Blutkultur, Knochenmark, Trachealsekret) bzw. auch einer weiteren Typisierung, oder anhand serologischer Testergebnisse.

Für den größten Teil der im Jahre 1995 an Brucellose Erkrankten wurde Br. melitensis als Infektionserreger ermittelt (Tab. 2). Allerdings lagen für fünf der insgesamt 34 Erkrankungen keine differenzierten Angaben zum Erreger vor (in vier Fällen Nachweis von $\mathrm{Br}$. abortus und $\mathrm{Br}$. melitensis, einmal Brucellen-IgM-Elisa). Für insgesamt sechs Erkrankungen, das entspricht $17,6 \%$, sind keine Angaben zur Diagnostik verfügbar.

\section{Vermutete Infektionsursache}

Hinweise auf eine Infektionsquelle liegen für immerhin 10 der 34 in die differenzierten Betrachtungen einbezogenen Brucellose-Erkrankungen nicht vor (Tab. 3). Die Auswertung der Angaben zu den übrigen 24 Erkrankungen gestaltet sich aufgrund vorliegender Doppelnennungen und in vielen Fällen tatsächlich auch mehrfach vorhandener Expositionsrisiken schwierig. Bei Patienten mit Tierkontakten, beispielsweise durch berufliche Tätigkeit (Tierpfleger,

\begin{tabular}{|c|c|c|c|c}
\hline Tierkontakt & $\begin{array}{c}\text { Frischmilch/ } \\
-k a ̈ s e\end{array}$ & Labortätigkeir & ohne Angabe & Summe \\
\hline $12\left(5^{*}\right)$ & $14\left(6^{*}\right)$ & $4(1 \%)$ & 10 & 40 \\
\hline $30 \%$ & $35 \%$ & $10 \%$ & $25 \%$ & $100 \%$ \\
\hline
\end{tabular}

* Doppelnennungen: 5 Fälle: Tierkontakte und Frischmilch/-käse-Verzehr 1 Fall: Frischmilch/-käse-Verzehr und Labortärigkejt 
Robert Koch - Institut

Fachgruppe Infektionsepidemiologie

Reichpietschufer 74-76

10785 Berlin

\section{Brucellose - Erhebungsbogen}

Meldewoche:

Jahr:

Bundesland:

Reg.-Bezirk:

ausgefüllt am:

Gesundheitsamt:

durch:

Meldeeingang im Gesundheitsamt:

Geschlecht: $\mathrm{m} \square$ w $\square$

Geb. Datum: bzw. Alter:

Deutscher $\square$ /Ausländer-Land: in Deutschland seit: als: Urlauber $\square \quad$ Erwerbstätiger $\square \quad$ Asylbewerber $\square \quad$ Student $\square$ sonstiger Grund:

erkrankt am: Arthralgie $\square$ sonstige Symptome:

Fieber $\square$ Kopfschmerz $\square \quad$ Mattigkeit $\square \quad$ Schweißausbrüche $\square$

erster Arztbesuch am:

hospitalisiert nein $\square$ ja $\square$ am: verstorben nein $\square$ ja $\square$ am:
Verdachtsdiagnose am: im:

diagnostische Sicherheit: klinisch $\square$ epidemiol. $\square$ Laborbestätigung $\square$ am: serologisch: 1:____ am: ___ ___ Erregernachweis aus: Erregernachweis aus:____ am: Erreger-Typ: Brucella abortus $\square$ melitensis $\square$ suis $\square$ canis $\square$ Biovar:

Infektionsquelle: vermutlich in Deutschland $\square$ im Ausland $\square$ Land: innerhalb der letzten 8 Wochen (evtl. noch früher) - von: von: __ bis: direkter Kontakt zu: Rindern $\square$ Ziegen $\square$ Schafen $\square$ anderen Tieren: beruflich $\square$ bzw. privat $\square$ Genuß unpasteurisierter Milch $\square$ bzw. von Frisch/Weichkäse aus unpasteurisierter Milch $\square$ andere mögliche Ursachen:

Umgebungsuntersuchungen: untersuchte Personen mit gleichem Risiko: pos. Nachweise:

\section{Bemerkungen:}


Schäfer, Schlachtung von Rindern), wird in mehreren Fällen (fünf Fälle) gleichzeitig auch Verzehr von Frischmilch bzw. -käse oder auch von rohem Fleisch außerhalb Deutschlands angegeben. Ebenso ist für einen weiteren $\mathrm{Er}$ krankungsfall nicht endgültig zu klären, ob der regelmäßige Verzehr von französischem Rohmilchkäse oder die berufliche Tätigkeit im mikrobiologischen Labor als Infektionsursache anzusehen sind.

Sicher scheint jedoch zu sein, daß unpasteurisierte Milch sowie Produkte aus Rohmilch, bevorzugt von Ziegen und Schafen, bei einer größeren Zahl von Brucellose-Erkrankungen des Jahres 1995 durchaus als infektionsverursachendes Lebensmittel anzusehen sind (für insgesamt 14 F ̈̈llle).

\section{Vermutetes Land der Infektion}

Den zahlenmäßig größten Anteil an den Brucellose-Erkrankungen des Jahres 1995 haben Erkrankungen, die in der Türkei erworben wurden (Tab. 4). Weitere Länder spielen für den Import von Brucellose-Erkrankungen nach Deutschland wahrscheinlich nur eine geringe Rolle. Eine genauere Betrachtung der einzelnen in Frage kommenden Infektionsursachen zeigt, daß beispielsweise Tierkontakte ihren Ursprung in vielen Fällen im Ausland haben (8 von insgesamt 13).

Hingewiesen werden soll an dieser Stelle auf eine Brucellose-Erkrankung $(\mathrm{Br}$. abortus), die eine deutschstämmige Aussiedlerin aus Kasachstan betrifft. (Ein weiterer Importfall aus diesem Land liegt bereits für 1996 vor. Eine Tierärztin erkrankte zwei Wochen nach ihrer Einreise aus Kasachstan (Br. melitensis).)

Alle Brucellosen, die im Jahre 1995 durch berufliche Tätigkeit bedingt waren, befinden sich unter den vermutlich in Deutschland erworbenen Erkrankungsfällen. Zu den 1995 gemeldeten Brucellosen gehören auch drei Fälle (zwei Fälle vermutlich in Deutschland

Tabelle 4: Vermutetes Land der Infektion für die Brucellose-Erkrankungen 1995

\begin{tabular}{c|c|c|c|c|c}
\hline Deutschland & Türkei & $\begin{array}{c}\text { Syrien/ } \\
\text { Jordanien }\end{array}$ & $\begin{array}{c}\text { weitere } \\
\text { Länder* }\end{array}$ & ohne Angabe & Summe \\
\hline 9 & 11 & 3 & 5 & 6 & 34 \\
\hline $26 \%$ & $32 \%$ & $9 \%$ & $15 \%$ & $18 \%$ & $100 \%$ \\
\hline
\end{tabular}

* Jeweils ein Fall aus Griechenland, Kasachstan, Kroatien, Marokko und Österreich. erworben, ein Fall in der Türkei), die bereits in früheren Jahren zu BrucelloseErkrankungen geführt hatten und deshalb im Sinne der Meldeverordnung keine Neuerkrankungen darstellen. Zukünftig können die Erkrankungsfälle im Rahmen der laufenden Sondererhebungen hierauf geprüft und ggf. nicht in die Statistik aufgenommen werden.

\section{Herkunft der Erkrankten}

Entsprechend den vermuteten Ländern, in denen die Infektion erfolgte, sind mehr ausländische als deutsche Bürger von Brucellose-Erkrankungen betroffen (Tab. 5). Diese sind zu einem großen Teil türkischer Herkunft (insgesamt 11 Erkrankte).

Verteilung der Erkrankungsfälle nach Altersgruppen und Geschlecht

Von den Brucellose-Erkrankungen 1995 sind vorwiegend Erwachsene betroffen. Insgesamt $70 \%$ aller Erkrankten befinden sich im Alter von 26 bis 65 Jahren. Sieben Patienten ( $21 \%$ ) gehören der Altersgruppe 0 bis 15 Jahre an. Zwei Patienten sind älter als 65 Jahre (6\%), von einem Patienten ist das Alter nicht bekannt (3\%). Betrachtet man die Verteilung nach Geschlecht der Erkrankten, so überwiegen männliche Patienten (21 männliche, 13 weibliche).

\section{Zusammenfassende Bewertung}

Brucellose-Erkrankungen als lebensmittelbedingte Infektionskrankheiten

Brucellose-Erkrankungen des Menschen gehören in Deutschland zu den eher selten auftretenden Infektionskrankheiten. In früheren Jahren wurde

\begin{tabular}{|c|c|c|c|}
\hline Deutsche & Ausländer* & ohne Angabe & Summe \\
\hline 11 & 15 & 8 & 34 \\
\hline $32 \%$ & $44 \%$ & $24 \%$ & $100 \%$ \\
\hline
\end{tabular}

* Davon: 11 x Türkei, 1 x Griechenland, 1 x Afrika, 1 x Kasachstan und 1 x arabische Länder.

der größte T'eil der Brucellose-Erkrankungen durch Tierkontakte erworben. Noch in den Jahren 1976/77 erkrankten vorzugsweise in der Landwirtschaft Tätige, Schlächter und Veterinäre, vor allem in den südlichen Bundesländern mit Weidewirtschaft [6]. Einen direkten Zusammenhang zwischen BrucelloseErkrankungen beim Tier und beim Menschen ergaben zu Beginn der achtziger Jahre erstellte Statistiken, nach denen die höchsten absoluten Erkrankungsziffern auf die Bundesländer entfielen, in denen auch Tierbrucellosen am häufigsten vorkamen [9].

Auch für die DDR traf zu, daß es sich bei den Erkrankungen an Brucellose vorwiegend um Kontaktinfektionen bei Beschäftigten in landwirtschaftlichen Betrieben handelte, wobei lebensmittelbedingte Infektionen wegen der Pasteurisierung der Milch zur damaligen Zeit praktisch keine Rolle mehr spielten [5]. Von den in den Jahren 1980 bis 1989 in diesem Gebiet insgesamt nur 25 gemeldeten Erkrankungen handelte es sich bei 17 um wahrscheinliche Berufsinfektionen, bei drei Fällen um importierte Erkrankungen und in zwei Fällen um Laborinfektionen. (Die Ursachen der drei restlichen Fälle blieben ungeklärt.)

Im Verlaufe der letzten Jahre kam es zu einer Veränderung des Charakters dieser Erkrankung - von der ehemals eng mit den Brucellose-Erkrankungen beim Tier verbundenen Erkrankung erfolgte ein Wandel zur vorwiegend durch Lebensmittel verursachten Infektionskrankheit. In Zusammenhang damit kam es auch zu einem anderen Verteilungsspektrum der Erregerarten. Wurde in den zusammenfassenden Berichten von Weise $[6,7]$ noch ein starkes Überwiegen der Bangschen Krankheit und nur seltenes Auftreten des Maltafiebers festgestellt, so ist heute ein größerer Anteil von Erkrankungen an Maltafieber und damit ein Überwiegen von Br. melitensis festzustellen.

Im Ergebnis der im damaligen Bundesgesundheitsamt durchgeführten Brucellaisolierungen und -biotypisierungen 
deutete sich die zunehmende Bedeutung von Infektionen durch $\mathrm{Br}$. melitensis bereits Mitte der achztiger Jahre an [10].

Die Tendenz des Überganges von früher vorwiegend durch Tierkontakte bedingten Erkrankungen zu lebensmittelbedingten Erkrankungen ist auch für andere Länder, insbesondere auch für $\mathrm{Ka}$ lifornien, beschrieben worden [11]. Der Anteil von Br. melitensis an allen bakteriologisch bestätigten Brucellose- $\mathrm{Er}$ krankungen beträgt dort ca. $79 \%$.

Heute wird ein großer Teil der in Deutschland registrierten BrucelloseErkrankungen aus anderen Ländern importiert. Hieran sind insbesondere türkische Bürger beteiligt, bei denen diese Erkrankungen in vielen Fällen im Anschluß an Heimataufenthalte auftreten. An den importierten Brucellose-Erkrankungen haben auch deutsche Touristen, die während ihrer Urlaubsreisen unpasteurisierte Milch bzw. Frischmilchprodukte, insbesondere Ziegenoder Schafskäse, verzehren, einen nicht geringen Anteil.

In verschiedenen Untersuchungen zeigte sich, daß die Lebensfähigkeit von Brucellen in Molkereiprodukten beträchtlich ist. So konnten in bestimmten ausländischen Weichkäsearten teilweise lebensfähige Brucellen nachgewiesen werden [12].

Die engen Kontakte zu südlichen Ländern, insbesondere des Mittelmeerraumes, durch in Deutschland lebende Bürger dieser Länder und durch intensiven Reiseverkehr begünstigen den Import der Brucellose nach Deutschland. In diesen Ländern ist der Erreger Br. melitensis relativ stark verbreitet.

Auch in England und Wales, wo in den Jahren 1992-1994 insgesamt 44 Labormeldungen über Brucellose-Infektionen beim Menschen berichtet wurden, ist für einen großen Teil dieser Erkrankungen die Infektionsquelle im Ausland zu finden [8]. Dabei handelte es sich in der Regel um Einzelfälle.

Die Bewertung des Spektrums der Erregerarten, d. h. die Einschätzung, welche Erreger gegenwärtig dominieren, gestal- tet sich anhand der Angaben auf den Erhebungsbögen äußerst schwierig. Ein großer Teil der Diagnostik, Schätzungen gehen von $85 \%$ der Brucellosen aus [13], beruht auf serologischen Bestimmungsmethoden, d. h. einem Antikörpernachweis im Patientenserum. Serologische Testverfahren sind gattungsspezifisch und gestatten keinen Rückschluß auf den verursachenden Erregertyp. Dieser läßt sich bisher nur über eine kulturelle Anzucht ermitteln.

Jedoch gestatten neuere serologische Testmethoden (beispielsweise ELISA) einen Nachweis auch von spezifischen IgM-Antikörpern, womit eine frische Erkrankung von chronischen Infektionen unterschieden werden kann.

Sondererhebungen für ausgewählte meldepflichtige Infektionskrankheiten

Die für das Jahr 1995 begonnenen Sondererhebungen von Brucellose-Erkrankungen zeigten, daß ein großes Interesse und eine große Bereitschaft zur Mitarbeit an epidemiologischen Untersuchungen vorhanden sind. So wurden 34 der 36 versandten Erhebungsbögen ausgefüllt zurückgegeben, teilweise nach Konsultation von Ärzten in Klinik und Praxen.

Leider liegen für einige Fälle zu Detailfragen keine bzw. nur unzureichende Angaben vor, für die in aller Regel Informationen verfügbar sein könnten. Beispielsweise blieben in sechs Fällen die Fragen zur Art der Diagnostik (zum Nachweis von Brucellen bzw. zum serologischen Nachweis) unbeantwortet. Ebenfalls liegen zum Herkunftsland der Erkrankten in acht Fällen keine Angaben vor. Auch für andere erbetene Informationen, wie die vermutete Infektionsursache sowie das Land der Infektion, waren konkrete Daten nicht immer zu ermitteln. In vielen Fällen ist es naturgemäßig schwierig, epidemiologisch relevante Zusammenhänge herzustellen und hierzu Angaben zu machen.

Insgesamt zeigten die begonnenen Einzelfall-Erfassungen jedoch, daß wertvolle Informationen zum Vorkommen bestimmter Infektionskrankheiten hier- mit gewonnen werden können. Mit der Erfassung weiterer Infektionskrankheiten auf diesem Wege wurde begonnen.

Literatur:

[1] Dorn, Ch., und Alban, G.: Biotypisierung von Brucellen am $B G A / B g V V$ in den Jahren 1990-94. Bundesgesundhbl. 39 (1996) 65-68.

[2] Moegle, H., Heizmann, W., Katz, P., und Botzenhart, K.: Bericht über eine Brucellamelitensis-Epidemie in Süddeutschland. Bundesgesundhbl. 28 (1985) 69-74.

[3] Statistisches Bundesamt: Gesundheitswesen, Fachserie 12, Reihe 2: Meldepflichtige Krankheiten. Wiesbaden (jährlich).

[4] Statistisches Bundesamt: Gesundheitswesen, Fachserie 12, Reihe 4: Todesursachen. Wiesbaden (jährlich).

[5] Pöhn, H. P., und Rasch, G.: Statistik meldepflichtiger übertragbarer Krankheiten. BGA-Schriften 5/93. München: MMV Medizin Verlag 1994.

[6] Weise, H. J.: Die aktuelle Bedeutung der Zoonosen im Rahmen meldepflichtiger Infektionskrankheiten des Menschen. Bundesgesundhbl. 20 (1977) 353-362.

[7] Weise, H. J.: Die meldepflichrigen Zoonosen in der Bundesrepublik Deutschland einschl. Berlin (West) 1970-1979. Bundesgesundhbl. 24 (1981) 395-403.

[8] CDR Weekly 5, 32 (1995) 151.

[9] Scheibner, E.: Brucellosevorkommen in der Bundesrepublik Deutschland einschl. Berlin (West) Stand: Juni 1981. Bundesgesundhbl. 24 (1981) 408-410.

[10] Protz, D.: Vorkommen von Brucella melitensis in der Bundesrepublik Deutschland unter Berücksichtigung sero-diagnostischer Besonderheiten. Bundesgesundhbl. 30 (1987) 55-60.

[11] Chomel, B. B., DeBess, E. E., Mangiamele, D. M., Reilly, K. F., Farver, T. B., Sun, R. K., and Barrett, L. R.: Changing Trends in the Epidemiology of Human Brucellosis in California from 1973 to 1992: A shift toward Foodborne Transmission. J. Infect. Dis. 170 (1994) 1216-1223.

[12] Großklaus, D.: Bekämpfung von Zoonosen. Bundesgesundhbl. 20 (1977) 363-370.

[13] Brucellosen. Erkennung und Behandlung. Merkblatt für Ärzte (Stand: 1996). Hrsg.: Bundesinstitut für gesundheitlichen Verbraucherschutz und Veterinärmedizin und Robert Koch-Institut, Bundesinstitut für Infektionskrankheiten und nicht übertragbare Krankheiten. Köln: Deutscher Ärzte-Verlag GmbH.

Anschrift der Verfasser

Dr. Gernot Rasch, Dr. Irene Schöneberg, Dr. Lothar Apitzsch und Ursula Menzel, Robert KochInstitut, Fachgebiet 411 »Meldepflichtige Krankheiten «, Postfach 650280,13302 Berlin 